\title{
The Stochastic Shapley Value for Coalitional Games with Externalities
}

\author{
Oskar Skibski ${ }^{\mathrm{a}, *}$, Tomasz P. Michalak ${ }^{\mathrm{a}, \mathrm{b}}$, Michael Wooldridge ${ }^{\mathrm{b}}$ \\ ${ }^{a}$ Institute of Informatics, University of Warsaw, Banacha 2, 02-097 Warszawa, Poland \\ ${ }^{b}$ Department of Computer Science, University of Oxford, Oxford, OX1 3QD, UK
}

\begin{abstract}
A long debated but still open question in the game theory literature is that of how to extend the Shapley Value to coalitional games with externalities. While previous work predominantly focused on developing alternative axiomatizations, in this article we propose a novel approach which centres around the coalition formation process and the underlying probability distribution from which a suitable axiomatization naturally follows. Specifically, we view coalition formation in games with externalities as a discrete-time stochastic process. We focus, in particular, on the Chinese Restaurant Process a well-known stochastic process from probability theory. We show that reformulating Shapley's coalition formation process as the Chinese Restaurant Process yields in games with externalities a unique value with various desirable properties. We then generalize this result by proving that all values that satisfy the direct translation of Shapley's axioms to games with externalities can be obtained using our approach based on stochastic processes.
\end{abstract}

Keywords: Cooperative games, Shapley Value, Externalities 2000 MSC: $91 \mathrm{~A} 12$

JEL: C71

\footnotetext{
*Corresponding author

Email addresses: oskar.skibski@mimuw.edu.pl (Oskar Skibski), tomasz.michalak@cs.ox.ac.uk (Tomasz P. Michalak), michael.woodlridge@cs.ox.ac.uk (Michael Wooldridge)
} 


\section{Introduction}

The problem of how to fairly divide a value obtained through cooperation is one of the most fundamental issues studied in coalitional game theory. It is relevant to a wide range of economic and social situations, from sharing the cost of a local wastewater treatment plant, through dividing the annual profit of a joint venture enterprise, to determining power in voting bodies. Assuming that the coalition of all the players (i.e. the grand coalition) forms, Shapley [1] proposed to remunerate the players by considering their marginal contributions to all coalitions they could possibly join. His division scheme, now called the Shapley Value, is the unique one satisfying the following four axioms: Efficiency - all payoff is distributed among the players; Null-Player - a player with no influence on payoffs receives nothing; Symmetry - symmetric players obtain the same payoff; and Additivity - the division scheme is additive over games.

However, Shapley's remarkable result holds only when one cooperative arrangement of players does not impose any externalities on any other cooperative arrangements. Such an assumption is clearly untenable in many practical economic situations of interest. For example, in an oligopolistic market, joint R\&D projects are meant to increase the competitive edge of cooperating companies [2]. Similarly, the extent of pollution reduction achieved by an international treaty depends not only on the signatories to the treaty, but also on similar agreements among non-participants [3]. In all such situations it is necessary to consider coalitions not as independent entities but rather coalitions embedded in coalition structures. Extending the Shapley Value to this richer setting has been a subject of ongoing debate in the literature for more than forty years. This issue is also the focus of our article.

Mathematically, the Shapley Value in games with no externalities is defined as the average marginal contribution of a player, taken over all possible permutations of players. It can be rationalized by the following deterministic coalition formation process proposed by Shapley [1, p. 39]. Assume that players create the grand coalition sequentially in a random order. A new player receives the payoff that equals his marginal contribution to the group of players that he joins. Now, the Shapley Value is the player's expected such payoff over all random orders (permutations) of players.

Extending the Shapley Value has turned out to be a challenging research problem because an obvious (or, as we will say, direct) translation of Shapley's axioms to games with externalities does not yield a unique value. The 
key problem is the Null-Player Axiom, as its direct translation to games with externalities is too weak to guarantee uniqueness.

A number of methods have been developed in the literature to address this issue. Some, such as Pham Do and Norde [4] and $\mathrm{Hu}$ and Yang [5], obtained uniqueness by proposing a stronger version of the original NullPlayer Axiom. Other contributors have moved increasingly further away from Shapley's original axiomatization by adding new axioms, and sometimes dropping some of the original ones. An example of such an approach is the work by Grabisch and Funaki [6], who used Markovian and Ergodic Axioms and modified the Symmetry and the Null-Player Axioms. Yet another method has been to build extensions to games with externalities relying on alternative axiomatizations of the original Shapley Value, such as Myerson's [7] balanced-contribution axiomatization or Young's [8] monotonicity axiomatization.

While previous works predominantly focused on axiomatizations for games with externalities, the extension of the Shapley Value proposed in this article centres around the coalition formation process and the underlying probability distribution from which a suitable axiomatization naturally follows. In particular, we view coalition formation in games with externalities as a discretetime stochastic process, in which the players leave the grand coalition one after another in a random order. Next, they either join one of the existing groups outside or form a completely new group, all with a certain probability. Each player receives the payoff that equals her marginal contribution to the coalition she left.

One of the fundamental stochastic processes studied in the literature is the Chinese Restaurant Process. This process and its variants have been used in a variety of applications ranging from modelling texts to genetics and functional genomics $[9,10,11,12,13]$. As we will show in this article, the Chinese Restaurant Process turns out to be of special interest also in the context of coalitional games with externalities. To gain some intuition behind it, let us consider the following fictional scenario. Let us imagine a Chinese restaurant with an infinite number of tables, each with an infinite number of seats. The first customer sits at the first table. Every new customer chooses a seat next to a customer that sits at one of the occupied tables, or a seat at the first unoccupied table, always with the same probability. Hence, the $k$-th customer chooses a table with $b$ customers with probability $\frac{b}{k}$ or chooses an unoccupied table with probability $\frac{1}{k}$. In our cooperative game context, each table represents a coalition of players and all the tables 
taken together represent a coalition structure that emerged as a result of a stochastic coalition formation process.

Our key results can be summarized as follows. We show that reformulating Shapley's coalition formation process as the Chinese Restaurant Process yields a unique value in games with externalities that has various desirable properties. In particular, it is a well-known value from the literature derived from two different axiomatizations: first, by Feldman [14] using axioms of Additivity, Symmetry, Carrier (which is a combination of the Null-Player Axiom and Efficiency), Per Capita Liability, and Per Capita Claim; next, by Macho-Stadler et al. [15] using the axioms of Efficiency, Null-Player, Additivity, Strong Symmetry and Similar Influence. ${ }^{1}$ In this article, using our stochastic process approach, we show that this value can be derived from the direct translation of Shapley's axioms to games with externalities, where we obtain uniqueness by strengthening the original Null-Player Axiom based on the Chinese Restaurant Process. In what follows, we refer to this value as the Stochastic Shapley Value.

Next, we extend the above result by considering a generalized version of the Chinese Restaurant Process in which the partition of players emerges with an arbitrary probability distribution. We prove that all values that satisfy the direct translation of Shapley's axioms to games with externalities can be obtained using such a generalized process. This means, in particular, that by choosing appropriate probability distributions we obtain the values by Pham Do and Norde [4], McQuillin [16], Bolger [17], Hu and Yang [5], and Myerson [18]. We also generalize the axiomatization by proving that the direct translation of Shapley's axiomatization-Efficiency, Symmetry, Additivity and the Null-Player Axiom strengthened using the generalized stochastic process - is enough to obtain uniqueness. This result yields, in particular, axiomatizations of these five values that are close to Shapley's original axiomatization.

The remainder of this article is organized as follows. In the next section, we present basic definitions and notation. In Section 3, building upon on the Chinese Restaurant Process, we derive and then axiomatize the Stochastic Shapley Value. In Section 4, we consider the generalization of this approach. Finally, in Section 5, we position our results among other works in the literature. Conclusions follow.

\footnotetext{
${ }^{1}$ See Section 5 for more details on both axiomatizations.
} 


\section{Preliminary Definitions}

Let $U=\{1,2, \ldots\}$ denote the universe of players. For a given set of players $N=\{1,2, \ldots, n\} \subset U$, a coalition, $S$, is any subset of $N$. A partition, denoted $P$, is any set of disjoint coalitions whose union is $N$. A pair $(S, P)$, where $P$ is a partition of $N$ and $S \in P$, is called an embedded coalition. The set of all partitions and the set of all embedded coalitions over $N$ are denoted by $\mathcal{P}(N)$ and $E C(N)$, respectively, or simply, $\mathcal{P}$ and $E C$ when the set of players is clear from the context.

A game (in partition-function form) is given by a function $v$ that associates a real number with every embedded coalition, i.e., $v: E C(N) \rightarrow \mathbb{R}$. A game has externalities if there is at least one coalition the value of which depends on the arrangement of outside players, that is, for at least one coalition $S$ there exist two partitions $P_{1}, P_{2}$ containing $S$ such that $v\left(S, P_{1}\right) \neq v\left(S, P_{2}\right)$. If this is not the case then we say that the game is with no externalities. Such games can be represented in the well-known characteristic-function form: $\hat{v}: 2^{N} \rightarrow \mathbb{R}$. The outcome of the game (or the value of the game) is a function $\varphi$ that, for a given game, associates a vector of $n$ real numbers, one for each player, i.e., $\varphi:(E C(N) \rightarrow \mathbb{R}) \rightarrow \mathbb{R}^{N}$. Thus, $\varphi_{i}(v)$ denotes the value $\varphi$ of player $i$ in game $v$.

We will often make use of the class of simple games $\left\langle e^{(S, P)}\right\rangle_{(S, P) \in E C}$ :

$$
e^{(S, P)}(S, P)=1 \text { and } e^{(S, P)}(\tilde{S}, \tilde{P})=0 \text { otherwise. }
$$

Thus, in a simple game $e^{(S, P)}$ only coalition $S$ in partition $P$ has non-zero payoff.

The set of all permutations of a set $S$ will be denoted by $\Omega(S)$. As is common in combinatorics, we identify permutation $\pi \in \Omega(S)$ with a corresponding ordering. Formally, $\pi$ can be understood as a function that assigns positions to players, i.e., a bijection $\pi: S \rightarrow\{\mathbb{1}, 2, \ldots,|\mathbb{S}|\}$ (note that we use text decoration to distinguish the set of positions from the set of players). We will denote the set of players that appear in permutation $\pi$ after $i$ by $C_{i}^{\pi}$, i.e., $C_{i}^{\pi} \stackrel{\text { def }}{=}\{j \mid \pi(j)>\pi(i)\}$. Permutation $\pi \in \Omega(S)$ with $i \notin S$ added at the end will be denoted $\pi_{+i}$; formally, $\pi_{+i} \in \Omega(S \cup\{i\})$ is defined as $\pi_{+i}(j)=\pi(j)$ for $j \in S$, and $\pi_{+i}(i)=|\mathbb{S}|+\mathbb{1}$. In addition, for $S=\{1,2, \ldots,|S|\}$, we define permutation $\pi_{i d} \in \Omega(S)$ as $\pi_{i d}(k)=\mathbb{k}$ for every $k \in S$.

We use a shorthand notation for set subtraction and set union operations: $N_{-S} \stackrel{\text { def }}{=} N \backslash S$ and $S_{+\{i\}} \stackrel{\text { def }}{=} S \cup\{i\}$. Often, we omit brackets and simply 
write $S_{+i}$. In this spirit, for partitions, we will write $P_{-i}$ to denote the partition from $\mathcal{P}(N \backslash\{i\})$ that emerges from $P \in \mathcal{P}(N)$ by excluding player $i$. Furthermore, to denote the partition obtained by the transfer of player $i$ to coalition $T$ in partition $P$, we introduce the following notation:

$$
\tau_{i}^{T}(P) \stackrel{\text { def }}{=} P \backslash\{P(i), T\} \cup\left\{P(i)_{-i}, T_{+i}\right\},
$$

where $P(i)$ denotes $i$ 's coalition in $P$. In particular, if $T=\emptyset$, then $i$ forms a singleton coalition $\{i\}$. The partition obtained from $P$ by the transfer of a group of players $S$ to a new coalition will be denoted $P_{[S]}$. Formally, $P_{[S]} \stackrel{\text { def }}{=}\{T \backslash S \mid T \in P\} \cup\{S\}$.

For an arbitrary function $f: N \rightarrow X, f(S)$ is the image of $S: f(S) \stackrel{\text { def }}{=}$ $\{f(i) \mid i \in S\}$, and $f(P)$ is a set of images of coalitions from $P: f(P) \stackrel{\text { def }}{=}$ $\{f(T) \mid T \in P\}$. Consequently, $f(S, P)$ is defined as $f(S, P) \stackrel{\text { def }}{=}(f(S), f(P))$. Consider a bijection $f: N \rightarrow N$. As game $v$ and value $\varphi$ are functions, both $f(v)$ and $f(\varphi)$ are function compositions: $(f(v))(S, P)=v(f(S, P))$ and $f\left(\varphi_{i}\right)=\varphi_{f(i)}$. Intuitively, the value of embedded coalition $(S, P)$ in game $f(v)$ equals the value of an embedded coalition obtained by replacing all players $i$ from $(S, P)$ with $f(i)$. For example, if $f$ exchanges 1 and 2 , then $f(v)(\{1\},\{\{1\},\{2,3\}\})=v(\{2\},\{\{2\},\{1,3\}\})$.

As is customary in the literature, we assume that the grand coalition, the coalition $N$ of all players in the game, forms. Thus, the value of the game distributes the value of the grand coalition among the players. For games with no externalities, Shapley [1] famously proved that there exists a unique payoff distribution scheme that satisfies four desirable axioms. We state them in a general form, i.e., using the notation for games with externalities, so that their definition will also hold in the remainder of this article:

- Efficiency - the entire available payoff is distributed among the players: $\sum_{i \in N} \varphi_{i}(v)=v(N,\{N\})$ for every game $v$;

- Symmetry - payoffs do not depend on the players' names: $\varphi(f(v))=$ $f(\varphi)(v)$ for every game $v$ and every bijection $f: N \rightarrow N$;

- Additivity ${ }^{2}$ - the sum of payoffs in two separate games equals the payoff in a combined game: $\varphi\left(\beta_{1} v_{1}+\beta_{2} v_{2}\right)=\beta_{1} \varphi\left(v_{1}\right)+\beta_{2} \varphi\left(v_{2}\right)$ for all the

\footnotetext{
${ }^{2}$ This formulation of Additivity is consistent with other works on extending the Shapley
} 
games $v_{1}, v_{2}$ and scalars $\beta_{1}, \beta_{2} \in \mathbb{R}$, where $\left(v_{1}+v_{2}\right)(S, P) \stackrel{\text { def }}{=} v_{1}(S, P)+$ $v_{2}(S, P)$ and $(\beta v)(S, P) \stackrel{\text { def }}{=} \beta \cdot v(S, P)$;

- Null-Player Axiom - the players that do not have an impact on the value of any coalition should get nothing: if for every $(S, P)$ such that $i \in S$, and every $T \in P \backslash S \cup\{\emptyset\}$ it holds that $v(S, P)-v\left(S_{-i}, \tau_{i}^{T}(P)\right)=$ 0 , then $\varphi_{i}(v)=0$, for every game $v$ and player $i \in N$.

We will refer to the above axioms as the direct translation of Shapley's axioms, or, simply, Shapley's axioms. The Shapley Value is defined by the following equation:

$$
S V_{i}(\hat{v}) \stackrel{\text { def }}{=} \frac{1}{|N| !} \sum_{\pi \in \Omega(N)} \hat{v}\left(C_{i}^{\pi} \cup\{i\}\right)-\hat{v}\left(C_{i}^{\pi}\right),
$$

which can be rewritten as:

$$
S V_{i}(\hat{v}) \stackrel{\text { def }}{=} \sum_{S \subseteq N, i \in S} \frac{(|S|-1) !(|N|-|S|) !}{|N| !}(\hat{v}(S)-\hat{v}(S \backslash\{i\})) .
$$

As a rationalization of this value, Shapley [1, p. 39] presented the coalition formation process (or the "bargaining procedure") that we restate as follows:

Shapley Value (SV): Assume that players leave the grand coalition in a random order. As a player leaves, he receives a payoff that equals his marginal contribution to the group of players that he left. Now, the Shapley Value is the expected outcome of the player's contributions over all orders (permutations).

Value to games with externalities $[17,15,16,19]$. We note, however, that in the original axiomatization Shapley used a weaker version of Additivity: $\varphi\left(v_{1}+v_{2}\right)=\varphi\left(v_{1}\right)+\varphi\left(v_{2}\right)$. In games with no externalities, it is enough to imply that the value is linear; thus, the payoff division does not depend on the unit it is calculated with (i.e., $\varphi(\beta v)=\beta \varphi(v)$ ). However, in games with externalities, the weaker version of Additivity combined with Shapley's other three axioms implies that the value can be scaled, but only by rational numbers (see [15] for details). While we are not aware of real-life applications in which irrational values of coalitions occur, for consistency with the literature, we allow irrational numbers in the function domain; thus, we strengthen Additivity by the linearity condition. However, we retain the name Additivity, as we feel this is a natural translation of this axiom to games with externalities. 
Note that in the original formulation of Shapley's coalition formation process, players enter rather than leave the grand coalition. We will see later on that the current formulation is more convenient for games with externalities. Moreover, note that, in a particular permutation, the above process is deterministic - each player has only one action, namely, leave the coalition - and we are not concerned with what happens outside. However, in games with externalities, an outside partition of players may influence the value of the coalition; hence, we need to account for the actions of players outside the coalition. In the next section, we use the theory of stochastic processes to approach this more complex situation.

\section{The Stochastic Shapley Value}

In this section, we extend the Shapley Value to games with externalities based on a stochastic process widely known in the literature as the Chinese Restaurant Process. We begin with the definition of the process and the resulting value. Next, we present its axiomatic characterization.

\subsection{The Chinese Restaurant Process and the value}

The Chinese Restaurant Process (CRP) is a discrete-time stochastic process the value of which at time $t=n$ is a partition of the set of numbers $\{\mathbb{1}, 2, \ldots, m\}$. The probability of each partition is defined as follows. At time $t=1$, partition $\{\mathbb{1}\}$ is obtained. At time $t=n$, the last element, $\mathrm{m}$, either forms a new block $k^{3}$ with probability $\frac{1}{n}$ or is added to one of the existing blocks with probability $\frac{b}{n}$, where $b$ is the size of this block. As already mentioned in the Introduction, this process can be illustrated with a fictional Chinese restaurant in which the first customer sits at the first table and every new customer either sits directly next to someone at one of the already occupied tables or at the first unoccupied table, where each of those seats is chosen with the same probability.

We construct the value for games with externalities based on the Chinese Restaurant Process by assuming that this stochastic process determines the way in which the grand coalition gradually decomposes. In particular:

\footnotetext{
${ }^{3}$ While in game theory elements of the partitions are called coalitions, in probability theory they are called blocks. We will use both terms depending on the context.
} 
Stochastic Shapley Value: Assume that the players leave the grand coalition one after another in a random order and divide themselves into groups outside. In the $k$-th step, a departing player:

- either joins one of the existing groups outside with probability $b / k$ where $b$ is the size of this group;

- or forms a new group with probability $1 / k$.

As the result of her leaving, the player is assigned a payoff that equals her (elementary) marginal contribution, i.e., the loss of a coalition she left. Now, the value of a player is her expected payoff over all $n$ ! orders.

Based on the above process, we obtain the value:

$S S V_{i}(v) \stackrel{\text { def }}{=} \frac{1}{|N| !} \sum_{\pi \in \Omega(N)} \sum_{P \in \mathcal{P}} \frac{\prod_{T \in P}(|T|-1) !}{|N| !}\left(v\left(C_{i}^{\pi} \cup\{i\}, P_{\left[C_{i}^{\pi} \cup\{i\}\right]}\right)-v\left(C_{i}^{\pi}, P_{\left[C_{i}^{\pi}\right]}\right)\right)$.

which is a well-known value in the literature that has been already derived with two different axiomatizations (see Section 5 for more details). Given that we derive this value from the stochastic process, we refer to it as the Stochastic Shapley Value.

Note that the term $\prod_{T \in P}(|T|-1) ! /|N|$ ! is the probability that partition $P$ will form based on the Chinese Restaurant Process. Note also that formula (3) is similar to formula (1) for the standard Shapley Value with the difference that we take not only an average over all permutations but also a weighted average over all partitions.

Let us look more closely at the process of decomposing the grand coalition that leads to the Stochastic Shapley Value. In particular, let us focus at time $t=|N \backslash S|$, when a certain group of players, $S$, have not left yet the (remainder of the) grand coalition and that players from $N \backslash S$ have already done so and formed partition $P \backslash S$. Now, let $i \in S$ be the next player to leave $S$. We observe that the move (or transfer) of $i$ may affect the value of $S$ in different ways and this depends on the destination of the transfer. In more detail, if $i$ leaves $S$ and joins an existing coalition $T \in P \backslash S$ then her elementary marginal contribution will equal $v(S, P)-v\left(S_{-i}, \tau_{i}^{T}(P)\right)$; hence, it may be different for different $T$. Furthermore, if $i$ creates a new coalition then her contribution will equal $v(S, P)-v\left(S_{-i}, \tau_{i}^{\emptyset}(P)\right)$. 
Following the Chinese Restaurant Process, we define the CRP-marginal contribution of player $i$ to coalition $S(i \in S)$ embedded in partition $P$ as the expected elementary marginal contribution of her transfer outside of $S$.

$$
\left(m c_{i}^{C R P}(v)\right)(S, P) \stackrel{\text { def }}{=} \frac{1}{|N|-|S|+1} \sum_{j \in N \backslash S \cup\{\emptyset\}}\left(v(S, P)-v\left(S_{-i}, \tau_{i}^{P(j)}(P)\right)\right),
$$

where we assume that $P(\emptyset)=\emptyset$. By gathering all permutations in which player $i$ has the same marginal contribution, we obtain an equivalent formula for the Stochastic Shapley Value - it sums over all coalitions not permutations:

$$
S S V_{i}(v) \stackrel{\text { def }}{=} \sum_{(S, P) \in E C, i \in S} \frac{\prod_{T \in P}(|T|-1) !}{|N| !}\left(m c_{i}^{C R P}(v)\right)(S, P),
$$

and which is the counterpart of formula (2) for the Shapley Value.

In the next section, we axiomatically characterize the Stochastic Shapley Value.

\subsection{Axiomatic characterization}

Let us consider how to translate Shapley's Null-Player Axiom to games with externalities. If we translate it directly (i.e. strictly), we will call a player a null player if she never has any effect on the value of any coalition. This means that all her transfers outside the coalition should not change the value of this coalition. In other words, all her elementary marginal contributions equal zero. However, we can also choose a less strict translation. In particular, we may require that the expected elementary marginal contribution of a null player is zero but this does not have to be the case for her elementary marginal contributions. This latter translation leads to the following definition of a null-player:

CRP-Null-Player Axiom - a player whose CRP-marginal contribution to the value of any coalition is zero should get nothing: if for every $(S, P) \in E C$ such that $i \in S$ holds $\left(m c_{i}^{C R P}(v)\right)(S, P)=$ 0 , then $\varphi_{i}(v)=0$ for every game $v$ and player $i \in N$.

The following theorem shows that replacing the Null-Player Axiom with the CRP-Null-Player Axiom in a direct translation of Shapley's axioms is enough to imply a unique value. Moreover, this value is the Stochastic Shapley Value. 
Theorem 1. The Stochastic Shapley Value is the only value that satisfies Efficiency, Symmetry, Additivity and the CRP-Null-Player Axiom.

Proof. For all the proofs, we refer the reader to the appendix. The proof of Theorem 1 can be found on p. 28.

We have just shown that reformulating Shapley's coalition formation process as the Chinese Restaurant Process yields a unique value in coalitional games with externalities. In the next section, we discuss how this approach can be generalized to a wider family of stochastic processes.

\section{Generalization}

We derived the Stochastic Shapley Value above by assuming that players who leave the grand coalition join other coalitions with the probabilities dictated by the Chinese Restaurant Process. In this section, we show that this approach can be generalized by considering arbitrary probability distributions over the partitions that emerge in the process of dissolving the grand coalition. Next, generalizing Theorem 1, we prove that each such stochastic process with an underlying arbitrary probability distribution over partitions yields an extension of the Shapley Value to games with externalities that satisfies the corresponding direct translation of Shapley's axioms. Moreover, all values that satisfy the direct translation can be obtained with this approach.

\subsection{Generalization of the process and the value}

We begin by formalizing a family of probability distributions $p$ on the set of partitions of $\mathbb{1}, 2, \ldots 4$

Let $p=\left(p_{1}, p_{2}, \ldots\right)$ be a family of probability distributions in which $p_{n}$ is a probability distribution on all the partitions of the set of numbers $\{\mathbb{1}, 2, \ldots, \mathrm{m}\}$. Formally, $p_{n}$ can be defined as a function $p_{n}: \mathcal{P}(\{\mathbb{1}, \ldots, \mathrm{m}\}) \rightarrow$ $\mathbb{R}$ that satisfies $\sum_{P \in \mathcal{P}(\{\mathbb{1}, \ldots, \mathrm{n}\})} p_{n}(P)=1$ and $0 \leq p_{n}(P) \leq 1$ for every $P \in$ $\mathcal{P}(\{\mathbb{1}, \ldots, \mathfrak{n}\})$. Next, we specify $p_{n}$ on a partition $P_{k} \in \mathcal{P}(\{\mathbb{1}, \ldots, \mathbb{k}\})$ as the sum of probabilities of the partitions that cover $P_{k}$, i.e., such partitions

\footnotetext{
${ }^{4}$ In probability theory, a probability distribution, or a family of distributions, on the set of partitions of $\mathbb{1}, 2, \ldots$ is called a partition model [20].
} 
that restricted to the set $\{\mathbb{1}, \ldots, \mathbb{k}\}$ equal $P_{k}$ (formally, $R$ covers $P_{k}$ when $\left.\{S \cap\{\mathbb{1}, \ldots, \mathbb{k}\} \mid S \in R\}=P_{k}\right)$ :

$$
p_{n}\left(P_{k}\right) \stackrel{\text { def }}{=} \sum_{\substack{R \in \mathcal{P}(\{\mathbb{1}, \ldots, \text {, }\}) \\ R \text { covers } P_{k}}} p_{n}(R) .
$$

Note that we do not assume any relationship between the probabilities of different partitions. In particular, a partition that results from renaming some or all of the elements may have a different probability than the original one. In other words, the probability of a partition may depend not only on the sizes of the blocks but also on elements within them. Furthermore, we make no assumption on the relationship between different probability distributions in a given family. In particular, the probability $p_{n}\left(P_{k}\right)$ may differ from the probability $p_{n+1}\left(P_{k}\right)$.

Having specified the family of probability distributions $p$, let us now introduce a corresponding generalized stochastic process. In particular, the family of stochastic processes based on $p$ will be called the p-Process family, or the $p$-Processes for short. For a given $n$, a $p_{n}$-Process yields a random partition from $\mathcal{P}(\{\mathbb{1}, \ldots, n\})$. At time $t=1$, partition $\{\mathbb{1}\}$ is obtained. At time $t=k+1$, assuming that partition $P_{k}$ has been formed by the previous elements, the next element is added to one of the blocks (or creates a new block) with probability $p_{n}\left(P_{k+1}\right) / p_{n}\left(P_{k}\right)$, where $P_{k+1}$ is the partition resulting from adding element $\mathbb{k}+\mathbb{1}$.

Considering a hypothetical case, when $P_{k}$ has been formed but $p_{n}\left(P_{k}\right)=$ 0 , it may seem that the probabilities at time $k+1$ are not well defined. However, we observe that this case never materializes $-P_{k}$ never forms because the addition that yields partition $P_{k}$ has zero probability. Furthermore, it is easy to check that the probabilities of the transfers sum up to one. This is because:

$$
p_{n}\left(P_{k}\right)=\sum_{T \in P_{k}} p_{n}\left(P_{k} \backslash T \cup(T \cup\{\mathbb{k}+\mathbb{1}\})\right)+p_{n}\left(P_{k} \cup\{\{\mathbb{k}+\mathbb{1}\}\}\right)
$$

holds for every $P_{k} \in \mathcal{P}(\{\mathbb{1}, 2, \ldots, \mathbb{k}\})$ and $k<n$.

Consider $k=n$. Although, formally, for $P_{n} \in \mathcal{P}(\{\mathbb{1}, 2, \ldots, \mathfrak{m}\})$, the values of the partitions of size $n+1$ on the right-hand side of formula (6) are not specified, for technical convenience, we will sometimes use the right-hand side of (6) assuming that it sums up to $p_{n}\left(P_{n}\right)$. 
Let us now define the value for games with externalities based on an arbitrary $p$-Process family. To this end, recall that if $S \subseteq N$ is an arbitrary subset of $N$ of size $k, P_{k} \in \mathcal{P}(S)$ is a partition of $S$, and $\pi \in \Omega(S)$ is a permutation of $S$, then $\pi\left(P_{k}\right)=\left\{\pi(S) \mid S \in P_{k}\right\}$ maps partition $P_{k}$ to the corresponding partition of set $\{\mathbb{1}, 2, \ldots, \mathbb{k}\}$. The same holds also if $\pi \in \Omega(N)$ is a permutation of all players with players from $P_{k}$ at the first $k$ positions. The coalition formation process based on the $p$-Process family that leads to the corresponding value is as follows:

The p-Process Shapley Value for $n=|N|$ players: Assume that players leave the grand coalition $N$ in a random order $\pi$ and divide themselves into groups outside. Assume that after $k(k<n-1)$ steps, partition $P_{k}$ has been formed (outside of the remainder of the grand coalition). In the $(k+1)$-th step, one player departs and joins one of the coalitions (or forms a new coalition) with probability $p_{n-1}\left(\pi\left(P_{k+1}\right)\right) / p_{n-1}\left(\pi\left(P_{k}\right)\right)$, where $P_{k+1}$ is the partition obtained by this transfer. As the result of her leaving, the player is assigned a payoff that equals her elementary marginal contribution, i.e., the loss of a coalition she left. Finally, in the $n$-th step, the last player, independently of which coalitions she joins, is assigned the remaining value of the grand coalition. Now, the $p$-Process Shapley Value is her expected payoff over all $n$ ! orders.

Note that we define the value for $n$ players based on probability distribution $p_{n-1}$, not $p_{n}$. This is because the elementary marginal contribution of the last player is always the same, no matter which outside coalition she chooses.

Formally, the $p$-Process Shapley Value is defined as follows:

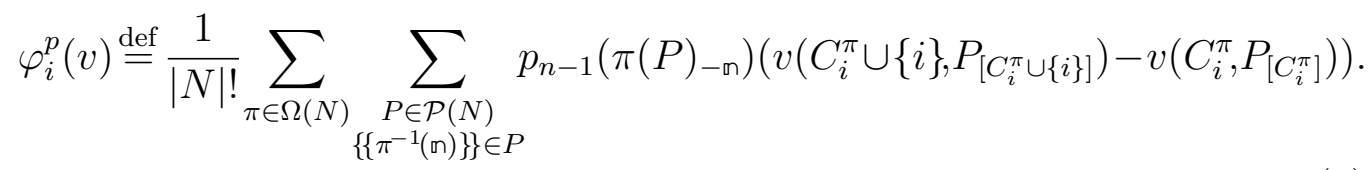

In the above formula we sum over all possible permutations and partitions of players. As the position of the last player does not matter, we assume for simplicity that she forms a singleton coalition. We will say that a value is obtained using the process approach for a family $p$ if it is defined using the above formula. 
Formula (7) can be rewritten as follows:

$$
\begin{array}{r}
\varphi_{i}^{p}(v)=\sum_{\substack{(S, P) \in E C \\
i \in S}} \frac{(|S|-1) !}{|N| !} \sum_{\pi \in \Omega(N \backslash S)} \sum_{T \in P \backslash S \cup\{\emptyset\}} p_{n-1}\left(\pi_{+i}\left(\tau_{i}^{T}(P) \backslash S_{-i}\right)\right) \\
\left(v(S, P)-v\left(S_{-i}, \tau_{i}^{T}(P)\right)\right),
\end{array}
$$

Note that for $S=\{i\}$ the above formula does not depend on the specific values of $p_{n-1}$ for partitions of size $n$ but only on their sum. This is because $v\left(S_{-i}, P\right)=0$ for every $P \in \mathcal{P}(N)$.

We observe that the Stochastic Shapley Value is the $p$-Process Shapley Value with $p_{n}^{C R P}$ defined as

$$
p_{n}^{C R P}(P) \stackrel{\text { def }}{=} \frac{\prod_{T \in P}(|T|-1) !}{|N| !},
$$

for every $P \in \mathcal{P}(N)$.

Although the coalition formation process makes sense only if values of $p$ are non-negative, from the mathematical point of view, the formulas for the $p$-Process Shapley Value are also well defined for negative values of $p$. If $p$ is allowed to take negative values, it is called a quasiprobability distribution. Formally, function $p_{n}: \mathcal{P}(\{\mathbb{1}, \ldots, \curvearrowleft\}) \rightarrow \mathbb{R}$ is a quasiprobability distribution, if $\sum_{P \in \mathcal{P}(\{\mathbb{1}, \ldots, \mathfrak{n}\})} p_{n}(P)=1$. For the sake of generality, we assume that the family $p$ also involves quasiprobability distributions. Hence, formally, the values of $p_{n}$ in (7) and (8) should be treated as weights instead of probabilities (note that formulas (5) and (6) also work for negative weights). A composition of such weights for a given permutation constitutes a weight of the resulting partition. The marginal contribution of a player in a given permutation is then calculated as the sum of all possible elementary marginal contributions multiplied by the corresponding weights of the resulting partitions. Nevertheless, we will typically refer to the values of $p_{n}$ as probabilities, as this term is more intuitive in the context of stochastic processes.

We are now ready to present the main technical result of this section. At face value, our approach to defining the values based on the stochastic processes may seem arbitrary; that is, there may exist other values that satisfy Shapley's axiomatization but that cannot be uniquely derived from the process approach. This is, however, not the case. The next theorem states that this approach encompasses all values that satisfy Shapley's axiomatization and exactly those. 
Theorem 2. A value can be obtained using the process approach for a family of quasiprobability distributions $p$ if and only if it satisfies Efficiency, Symmetry, Additivity and the Null-Player Axiom.

Proof. See the appendix, p. 28. We observe that, in the above theorem, it is not possible to weaken Additivity to the form $\varphi\left(v_{1}+v_{2}\right)=\varphi\left(v_{1}\right)+\varphi\left(v_{2}\right)$. For instance, Macho-Stadler et al. [15] provided an example of a value which satisfies Efficiency, Symmetry, the Null-Player Axiom and a weaker version of Additivity. However, this value violates homogeneity $(\varphi(\beta v)=\beta \varphi(v))$ which is clearly satisfied by all values obtained using $p$-Processes.

While Theorem 2 holds for quasiprobability distributions, we will now argue that if one is interested in monotonic values, then one must limit oneself only to $p$ with non-negative weights. In more detail, the property of monotonicity says that: ${ }^{5}$

(Weak) Monotonicity - an increase of a player's contributions does not decrease her payoff: if $v_{1}\left(S_{+i}, \tau_{i}^{S}(P)\right)-v_{1}(S, P) \geq$ $v_{2}\left(S_{+i}, \tau_{i}^{S}(P)\right)-v_{2}(S, P)$ holds for every $(S, P) \in E C$, such that $i \notin S$, then $\varphi_{i}\left(v_{1}\right) \geq \varphi_{i}\left(v_{2}\right)$.

We show now that the following holds:

Theorem 3. A value can be obtained using the process approach for a family of probability distributions $p$ if and only if it satisfies Efficiency, Symmetry, Additivity, the Null-Player Axiom, and (Weak) Monotonicity.

Proof. See the appendix, p. 31.

Having presented the generalization of the process and the value, in the next section we generalize the axiomatization.

\subsection{Generalization of the axiomatization: marginality approach}

In this section we generalize our axiomatization of the Stochastic Shapley Value from Section 3 by proposing a universal approach for axiomatizing

\footnotetext{
${ }^{5}$ This formulation of monotonicity agrees with definitions proposed by Macho-Stadler et al. [15] and De Clippel and Serrano [21].
} 
direct extensions of the Shapley Value to games with externalities. Broadly speaking, this approach, which we call the marginality approach, consists of strengthening of the Null-Player Axiom by considering a specific definition of marginal contribution. We will show that the marginality approach is strictly related to our general approach of obtaining extensions of the Shapley Value based on the $p$-Process as introduced in the previous section.

We start by generalizing the definition of the marginal contribution in games with externalities which now can take a form of any affine combination (i.e., linear combination with weights that sum up to one) of elementary marginal contributions:

$$
\left(m c_{i}^{\alpha}(v)\right)(S, P) \stackrel{\text { def }}{=} \sum_{T \in P \backslash S \cup\{\emptyset\}} \alpha_{i}\left(S_{-i}, \tau_{i}^{T}(P)\right)\left[v(S, P)-v\left(S_{-i}, \tau_{i}^{T}(P)\right)\right],
$$

where $\alpha_{i}:\{(S, P) \in E C \mid i \notin S\} \rightarrow \mathbb{R}$ denotes weights of a given transfer under the assumption that weights are symmetric (i.e., $\alpha_{i}(S, P)=$ $\alpha_{f(i)}(f(S), f(P))$ for every bijection $f: N \rightarrow N$ and $(S, P) \in E C$ such that $i \notin S$ ), and normalized (i.e., $\sum_{T \in P \backslash S \cup\{\emptyset\}} \alpha_{i}\left(S_{-i}, \tau_{i}^{T}(P)\right)=1$ for every $(S, P) \in E C$ such that $i \in S)$.

Having defined the marginal contribution parameterized by $\alpha$, let us now consider the corresponding definition of the Null-Player Axiom. In particular:

$\alpha$-Null-Player Axiom - players that do not contribute to the value of any coalition should get nothing: if for every $(S, P) \in E C$ such that $i \in S$ holds $\left(m c_{i}^{\alpha}(v)\right)(S, P)=0$, then $\varphi_{i}(v)=0$ for every game $v$ and player $i \in N$.

We observe that particular axiomatizations of the extensions of the Shapley Value to games with externalities can be seen as instances of the marginality approach - these concern the axiomatizations of the Bolger value [17], the externality-free value [4], the Hu-Yang value [5], and the McQuillin value [19]. In the next theorem, we generalize all these results from the literature and prove that Shapley's original axioms of Efficiency, Symmetry, Additivity and the parameterized Null-Player Axiom always yield a unique extension to games with externalities. Moreover, we show that this value can also be obtained using the $p$-Process and that for every monotonic $p$-Process Shapley Value, there exists an $\alpha$ such that Shapley's $\alpha$-parameterized axiomatization uniquely implies it. This also holds for almost all non-monotonic $p$-Process Shapley Values. 
Theorem 4. There exists a unique value that satisfies Efficiency, Symmetry, Additivity and the $\alpha$-Null-Player Axiom for every $\alpha$, and this value is the $p^{\alpha}$ Process Shapley Value, where $p^{\alpha}$ is defined as follows:

$$
p_{n}^{\alpha}(P)=\prod_{i \in\{1, \ldots, n\}} \alpha_{i}\left(\{i+1, \ldots, n, n+1\},\left(\pi_{i d}^{-1}(P) \cup\{n+1\}\right)_{[\{i+1, \ldots, n, n+1\}]}\right),
$$

for every $P \in \mathcal{P}(\{\mathbb{1}, \ldots, \mathrm{n}\})$. Moreover, for quasiprobability distribution $p$, there exists an $\alpha$ such that the p-Process Shapley Value is the value resulting from $\alpha$-parameterized Shapley's axiomatization if and only if $p$ satisfies zeroconsistency, i.e.,

$$
\sum_{\pi \in \Omega(\{\mathbb{1}, \ldots, \mathbb{k}-\mathbb{1}\})} p_{n}\left(\pi\left(\left(P_{k}\right)_{-\mathbb{k}}\right)\right)=0 \Rightarrow \sum_{\pi \in \Omega(\{\mathbb{1}, \ldots, \mathbb{k}-\mathbb{1}\})} p_{n}\left(\pi_{+\mathbb{k}}\left(P_{k}\right)\right)=0 .
$$

for every $n, k \leq n$, and $P_{k} \in \mathcal{P}(\{\mathbb{1}, \ldots, \mathbb{k}\})$.

Proof. See the appendix, p. 32. We note that, although we assumed a stronger definition of Additivity, our proof is based only on the weaker version, i.e., $\varphi\left(v_{1}+v_{2}\right)=\varphi\left(v_{1}\right)+\varphi\left(v_{2}\right)$, which does not require linearity. Consequently, the theorem also holds for the weaker version of Additivity.

\subsection{Other values in the process approach}

We are aware of five other values in the literature that satisfy the direct translation of Shapley's axioms to games with externalities. We proved in Section 4.1 that all those values can be obtained using the process approach. In this section, we show details of how this can be done and discuss the properties that those values satisfy.

We will consider two important properties of probability distributions: exchangeability and consistency. They characterize the necessary conditions for the famous theorem of de Finetti [22]. A probability distribution is exchangeable if relabelling elements does not change the distribution of the partition. Thus, the probability of the partition depends only on the sizes of the blocks, and not on their members. Formally, $p_{n}(P)=p_{n}(f(P))$ for every $P \in \mathcal{P}(\{\mathbb{1}, \ldots, \mathfrak{m}\})$ and bijection $f:\{\mathbb{1}, \ldots, \mathfrak{n}\} \rightarrow\{\mathbb{1}, \ldots, \mathfrak{m}\}$. Furthermore, a family of probability distribution $p=\left(p_{1}, p_{2}, \ldots\right)$ is (Kolmogorov) consistent (or projective) if $p_{n}\left(P_{k}\right)=p_{k}\left(P_{k}\right)$ for every $P_{k} \in \mathcal{P}(\{\mathbb{1}, 2, \ldots, k\}$ ) and $k<n$. Thus, the sum of probabilities according to distribution $p_{n}$ of 
the partitions that cover $P_{k}$ (i.e., partitions that are obtained by inserting elements $\{\mathbb{k}+\mathbb{1}, \ldots, \mathbb{m}\}$ into partition $\left.P_{k}\right)$ is equal to the probability of $P_{k}$ according to distribution $p_{k}$.

Bolger [17] was chronologically the first to propose a definition of marginal contribution in games with externalities. In particular, Bolger argued that, for a given embedded coalition, all transfers should have the same weight, i.e., $\alpha_{i}^{B}(S, P)=\frac{1}{\left|\tau_{i}^{S}(P)\right|}$. Next, Bolger derived his value using Young's axiomatization based on the Marginality Axiom. Using Theorem 4, we get the family of probability distribution for the Bolger value, $p^{B}=\left(p_{1}^{B}, p_{2}^{B}, \ldots\right)$, defined as follows:

$$
p_{n}^{B}(P)=\prod_{\mathbb{k} \in\{\mathbb{1}, \ldots, \mathfrak{m}\}} \frac{1}{\mid P_{[\{\mathbb{k}, \mathbb{k}+\mathbb{1}, \ldots, \mathfrak{m}\}]}} .
$$

This family of probability distribution is consistent, but it is not exchangeable, since $p_{n}^{B}(\{\{1\},\{2,3\}\})=1 / 6 \neq 1 / 4=p_{n}^{B}(\{\{1,2\},\{3\}\})$.

Two further values are dual. The externality-free value was proposed by Pham Do and Norde [4], who defined the marginal contribution as this elementary marginal contribution in which a player creates a new coalition. To obtain a unique value, the authors used Shapley's $\alpha$-parameterized axiomatization. This uniqueness result is a special case of Theorem 4. Later on, De Clippel and Serrano [21] derived the same value using Young's axiomatization (which is a special case of the result by Fujinaka - see Section 5). The externality-free value can be obtained through the process approach with the family of probability distributions, $p^{\text {free }}=\left(p_{1}^{\text {free }}, p_{2}^{\text {free }}, \ldots\right)$, defined as follows:

$$
p_{n}^{\text {free }}(P)= \begin{cases}1 & \text { if } P=\{\{\mathbb{k}\} \mid \mathbb{k} \in\{\mathbb{1}, \ldots, \mathbb{n}\}\} \\ 0 & \text { otherwise. }\end{cases}
$$

McQuillin [16] studied not only how to extend the Shapley Value to games with externalities but also how to combine such an extension with the Owen generalization to an a-priori given coalition structure [23]. His analysis led to two axioms, called Rules of Generalization and Recursion that, combined with Weak Monotonicity and Shapley's axioms, imply a unique value. The marginality-based axiomatization of the McQuillin value was later derived by Skibski [19]. This result is a special case of Theorem 4. In particular, the definition of marginal contribution by Skibski can be generalized to the 
family of probability distributions, $p^{\text {full }}=\left(p_{1}^{\text {full }}, p_{2}^{\text {full }}, \ldots\right)$ :

$$
p_{n}^{\text {full }}(P)= \begin{cases}1 & \text { if } P=\{\{\mathbb{1}, \ldots, \mathfrak{n}\}\} \\ 0 & \text { otherwise }\end{cases}
$$

In the $p$-Process interpretation of the externality-free value, a player always forms a new coalition. In contrast, in the case of the McQuillin value, any player leaving (the remainder of) the grand coalition always joins the only existing coalition outside. Both families of probability distributions, $p^{\text {free }}$ and $p^{\text {full }}$, are consistent and exchangeable. However, they contain zero probabilities. In other words, the McQuillin value and the externality-free value take into consideration values of only some embedded coalitions.

$\mathrm{Hu}$ and Yang [5] defined a specific measure of a player's contribution to each partition, and argued that the value of a player should be an average such contribution over all partitions, each taken with the same probability. This value can be obtained using the process approach with family $p^{H Y}=$ $\left(p_{1}^{H Y}, p_{2}^{H Y}, \ldots\right)$ defined as follows:

$$
p_{n}^{H Y}(P)=\frac{|P|+1}{|\mathcal{P}(\{1, \ldots, n, n+1\})|} .
$$

This family of probability distributions is exchangeable, but it is not consistent. In particular, $p_{2}^{H Y}(\{\{1\},\{2\}\})=1 / 2 \neq 3 / 5=p_{3}^{H Y}(\{\{1\},\{2\}\})$.

Finally, we discuss the value proposed by Myerson [18] which is based on the concept of a carrier. In more detail, we say that a set $C$ is a carrier if the value of any embedded coalition is determined by a partition of players from $C$. Now, the Carrier Axiom implies that, if $C$ is a carrier, then the payoff of the grand coalition is divided between players from $C$. Given this, Myerson showed that there exists a unique value that satisfies Symmetry, Additivity and the Carrier Axiom. Naturally, since the Myerson value satisfies all four of Shapley's axioms, then, based on Theorem 2, it can be derived using the process approach. However, Myerson's Carrier-based axiomatization is far from the process (or marginality) analysis and results in a complex set of quasi-probabilities with negative values that do not have any of the properties discussed above. For instance, for $N=\{1,2,3\}$, the formula for the Myerson value implies that $p_{2}(\{\{\mathbb{1}, 2\}\})=-1$ and $p_{2}(\{\{\mathbb{1}\},\{2\}\})=2$. 


\section{Related Work}

In the previous section, we presented results concerning all values that satisfy the direct translation of Shapley's axioms. In this section, we first discuss two alternative axiomatizations of the Stochastic Shapley Value. Then, we consider all the values that do not satisfy the direct translation of Shapley's axioms.

Two different axiomatizations from the literature yield the value that we termed the Stochastic Shapley Value. In the first work, Feldman [14] characterized the value with Additivity, Symmetry, Carrier (which is a combination of the Null-player Axiom and Efficiency), Per Capita Liability, and Per Capita Claim. Briefly speaking, Per Capita Liability states that the liabilities of any two coalitions relative to another coalition are equal on a per capita basis, and Per Capita Claim, that the claims of all coalitions are equal on a per capita basis.

In the second work, Macho-Stadler et al. [15] derived their value (that is homogeneous with the Stochastic Shapley Value) using Shapley's axioms together with Strong Symmetry and Similar Influence. Intuitively, Strong Symmetry requires that the value of any coalition has a symmetric influence on the payoffs of outside players, or, to put it differently, that a change in the value of an embedded coalition has a symmetric effect on all other players in the coalition structure. Similar Influence considers two embedded coalitions. In the first one, $i$ and $j$ appear as two singletons $\{i\}$ and $\{j\}$, and, in the second one, as the coalition $\{i, j\}$. Then, the Similar Influence axiom states that if we exchange the values of these two coalitions then $i$ 's and $j$ 's payoffs should not change.

Until now, we have focused on values that satisfy the direct translation of Shapley's axioms. In the remainder of this section, we consider those that do not. We begin with the work by Fujinaka [24] who was the first to propose a general formula for the marginal contribution as the affine combination of elementary marginal contributions. The author proved that Young's axiomatization parameterized by any weights $\alpha$ implies a unique value. Our Theorem 4 is the equivalent of Fujinaka's result but for Shapley's axiomatization.

Albizuri et al. [25] argued that, in a game with externalities, a coalition should be evaluated by the set of values it has, regardless of which partitions these values correspond to. This principle they called Embedded Coalition Anonymity. The authors combined this principle with The Oligarchy Axiom 
and three of Shapley's original axioms: Efficiency, Additivity and Symmetry. The resulting value can be derived as the Shapley Value for the game with no externalities that is calculated by assigning to every coalition an arithmetic average of all its values in games with externalities. Although, at first, it seems like a special case of the average approach, the weights violate a condition that is necessary to satisfy the Null-Player Axiom (see Theorem 1 in $[15])$.

In the Chinese Restaurant Process and, more generally, in the $p$-Process family, all players leave the grand coalition one by one. Grabisch and Funaki [6] proposed a different approach. In particular, as a starting point for their process, they took the partition containing only singletons. Next, they considered all possible sequences of players' mergers that lead to the grand coalition. The marginal contribution of each player is the effect of merging the player with a coalition. If a player enters some coalition alone, he is rewarded with the whole change of its value, i.e., with the marginal contribution; but if she is already a part of a coalition that merges with another one, Grabisch and Funaki argue that the change of the value of the coalition they merge with should be divided equally between her and other members of the coalition. This contradicts the Null-Player Axiom, as a null-player is rewarded with a payoff even though the coalition without her would cause the same impact on the merged coalition. Finally, we note that the work by Grabisch and Funaki is the only other work in the literature that extends the Shapley Value to games with externalities by focusing on the process (a deterministic one, in their case).

Finally, let us address the concepts proposed by Maskin [26] and Hafalir [27]. Both authors discarded the assumption that the grand coalition forms and proposed to divide the payoff of the optimal coalition structure. Maskin studied the coalition formation process and proposed an axiomatic characterization of the value yielded by this process. Conversely, Hafalir proposed a mechanism that implements a unique payoff division and provided axiomatization based on the idea of efficient-cover. These ideas, although interesting, lead to an axiomatization that is significantly different from Shapley's.

\section{Conclusions}

We were concerned in this article with the issue of deriving the extension

of the Shapley Value to games with externalities. We proved that this can 
be achieved by reformulating Shapley's coalition formation process as the stochastic process called the Chinese Restaurant Process which yields a wellknown value from the literature to which, in the context of our article, we refer as the Stochastic Shapley Value. We showed that this value satisfies the direct translation of Shapley's axioms to games with externalities, where the uniqueness is obtained by strengthening the original Null-Player Axiom based on the Chinese Restaurant Process.

Next, we extended the above result by considering a generalized version of the Chinese Restaurant Process and proved that all values that satisfy the direct translation of Shapley's axioms to games with externalities can be obtained using this approach. We also generalized the axiomatization and showed that the direct translation of Shapley's axiomatization with the NullPlayer Axiom strengthened using the generalized process is enough to obtain uniqueness. These results encompass as special cases the following values from the literature: Pham Do and Norde [4], McQuillin [16], Bolger [17], Hu and Yang [5], and Myerson [18].

Our work can be extended in various directions. Since our results combined with those of Fujinaka [24] show that Young's and Shapley's axiomatization in games with externalities are equivalent, it would be very interesting to study their relationship to Myerson's axiomatization [7]. This well-known axiomatization of the Shapley Value in games with no externalities is based on the principle of Balanced Contributions. Interestingly, in games with externalities, it can easily be shown that there exist values that satisfy Shapley's axioms but do not satisfy the principle of Balanced Contributions. Another interesting question is how the Stochastic Shapley Value can be implemented. Finally, we are keen to study computational properties of the Stochastic Shapley Value against other values in the literature.

\section{Acknowledgements}

Oskar Skibski was supported by the JSPS KAKENHI Grant (24220003). Tomasz Michalak and Michael Wooldridge were supported by the European Research Council under Advanced Grant 291528 ("RACE"). This work was also supported by the Polish National Science Centre Grant DEC-2013/09/D/ST6/03920. We would like to thank Andrzej Szalas for various comments and suggestions, and Jordan Summers-Young for careful proof reading of this article. 


\section{References}

[1] L. S. Shapley, A value for n-person games, in: H. Kuhn, A. Tucker (Eds.), Contributions to the Theory of Games, Vol. II, Princeton University Press, 1953, pp. 307-317.

[2] S.-S. Yi, Endogenous formation of economic coalitions: A survey on the partition function approach, in: The Endogenous Formation of Economic Coalitions, Edward Elgar, London, UK, 2003, pp. 80-127.

[3] M. Finus, New developments in coalition theory: An application to the case of global pollution, in: L. Marsiliani, M. Rauscher, C. Withagen (Eds.), Environmental Policy in an International Perspective, Kluwer Academic Publishers, Dordrecht, Holland, 2003, pp. 19-49.

[4] K. H. Pham Do, H. Norde, The Shapley value for partition function form games, International Game Theory Review 9 (02) (2007) 353-360.

[5] C.-C. Hu, Y.-Y. Yang, An axiomatic characterization of a value for games in partition function form, SERIEs 1 (4) (2010) 475-487.

[6] M. Grabisch, Y. Funaki, A coalition formation value for games with externalities, Tech. Rep. b08076, Universite Pantheon-Sorbonne (Paris 1), Centre d'Economie de la Sorbonne (2008).

[7] R. B. Myerson, Conference structures and fair allocation rules, International Journal of Game Theory 9 (1980) 169-82.

[8] H. P. Young, Monotonic solutions of cooperative games, International Journal of Game Theory 14 (2) (1985) 65-72.

[9] R. M. Neal, Markov chain sampling methods for Dirichlet process mixture models, Journal of Computational and Graphical Statistics 9 (2) (2000) 249-265.

[10] D. M. Blei, T. L. Griffiths, M. I. Jordan, J. B. Tenenbaum, Hierarchical topic models and the nested chinese restaurant process, in: Advances in Neural Information Processing Systems, MIT Press, 2004, p. 2003.

[11] Z. S. Qin, Clustering microarray gene expression data using weighted Chinese restaurant process, Bioinformatics 22 (16) (2006) 1988-1997. 
[12] E. P. Xing, M. I. Jordan, R. Sharan, Bayesian haplotype inference via the Dirichlet process, Journal of Computational Biology 14 (3) (2007) 267-284.

[13] W. J. Ewens, The sampling theory of selectively neutral alleles, Theoretical Population Biology 3 (1) (1972) 87-112.

[14] B. E. Feldman, Bargaining, coalition formation, and value, Ph.D. thesis, State University of New York at Stony Brook (1996).

[15] I. Macho-Stadler, D. Pérez-Castrillo, D. Wettstein, Sharing the surplus: An extension of the Shapley value for environments with externalities, Journal of Economic Theory 135 (1) (2007) 339-356.

[16] B. McQuillin, The extended and generalized shapley value: Simultaneous consideration of coalitional externalities and coalitional structure, Journal of Economic Theory 144 (2) (2009) 696-721.

[17] E. M. Bolger, A set of axioms for a value for partition function games, International Journal of Game Theory 18 (1) (1989) 37-44.

[18] R. B. Myerson, Graphs and cooperation in games, Mathematical Methods of Operations Research 2 (3) (1977) 225-229.

[19] O. Skibski, Steady marginality: A uniform approach to Shapley value for games with externalities, in: Proceeding of 4th Symposium on Algorithmic Game Theory, Vol. 6982 of LNCS, Springer, 2011, pp. 130-142.

[20] P. McCullagh, Random permutations and partition models, in: International Encyclopedia of Statistical Science, Springer, 2011, pp. 11701177 .

[21] G. De Clippel, R. Serrano, Marginal contributions and externalities in the value, Econometrica 76 (6) (2008) 1413-1436.

[22] F. Lehner, Cumulants in noncommutative probability theory IV. noncrossing cumulants: De Finetti's theorem and -inequalities, Journal of Functional Analysis 239 (1) (2006) 214-246.

[23] G. Owen, Values of games with a priori unions, in: Mathematical Economics and Game Theory, Vol. 141 of LNEMS, Springer, Berlin, 1977, pp. $76-88$. 
[24] Y. Fujinaka, On the marginality principle in partition function form games, unpublished manuscript (2004).

[25] M. J. Albizuri, J. Arin, J. Rubio, An axiom system for a value for games in partition function form, International Game Theory Review 7 (01) (2005) 63-72.

[26] E. Maskin, Bargaining, coalitions, and externalities, presidential address of the Econometric Society (2003).

[27] I. E. Hafalir, Efficiency in coalition games with externalities, Games and Economic Behavior 61 (2) (2007) 242-258. 


\section{APPENDIX: Proofs of Theorems}

We start by proving two lemmas (Lemmas 1 and 2) which are crucial in proving Theorems 1, 2, 3, and 4 .

Lemma 1. There exists a unique value that satisfies Efficiency, Additivity, Symmetry and the $\alpha$-Null-Player Axiom for arbitrary weights $\alpha$.

Proof. Let us consider the class of simple games $e^{(S, P)}$. Since this class forms the basis of the game space, every game can be defined as a linear combination of games $e^{(S, P)}: v=\sum_{(S, P) \in E C} v(S, P) \cdot e^{(S, P)}$. Based on the weaker version of Additivity $\left(\varphi\left(v_{1}+v_{2}\right)=\varphi\left(v_{1}\right)+\varphi\left(v_{2}\right)\right)$, we have that

$$
\varphi(v)=\sum_{(S, P) \in E C} \varphi\left(v(S, P) \cdot e^{(S, P)}\right) ;
$$

hence, it is enough to prove that the axioms imply a unique value in a simple game, $e^{(S, P)}$, multiplied by a scalar. For this purpose, we will use the reverse induction on the size of $S$. In particular, we will show that the value of game $e^{(S, P)}$ can be calculated from the values of simple games for bigger coalitions $e^{(\tilde{S}, \tilde{P})}$, where $|\tilde{S}|>|S|$. Our base case, when $|S|=|N|$, comes from the Efficiency and Symmetry: $\varphi_{i}\left(c \cdot e^{(N,\{N\})}\right)=\frac{c}{|N|}$ for every $i \in N$.

First, let $(S, P)$ be an embedded coalition and let us assume that $i \notin S$. We consider game $\tilde{v}$ that is a combination of two simple games:

$$
\tilde{v}=c \cdot\left(\alpha_{i}(S, P) \cdot e^{\left(S_{+i}, \tau_{i}^{S}(P)\right)}+e^{(S, P)}\right) .
$$

It is easy to observe that the player $i$ 's marginal contribution to $\left(S_{+i}, \tau_{i}^{S}(P)\right)$ equals zero, as it is also the case with all other marginal contributions. Thus, from the $\alpha$-Null-Player Axiom we have that $\varphi_{i}(\tilde{v})=0$, and from Additivity we have that for $i \notin S$ it holds that:

$$
\varphi_{i}\left(c \cdot e^{(S, P)}\right)=-\varphi_{i}\left(c \cdot \alpha_{i}(S, P) \cdot e^{\left(S_{+i}, \tau_{i}^{S}(P)\right)}\right) .
$$

Now, let us assume otherwise, i.e., that $i \in S$ and $|S|<|N|$ (recall that we have already considered simple game $\left.e^{(N,\{N, \emptyset\})}\right)$. We have that $v(N,\{N, \emptyset\})=$ 0 . From Efficiency, we can evaluate the sum of payoffs of players from $S$ as the opposite value to the sum of payoffs of outside players $\left(-\sum_{j \notin S} \varphi_{j}\left(c \cdot e^{(S, P)}\right)\right)$. This sum, in turn, can be calculated with formula (.1). Now, based on 
Symmetry, all players from $S$ divide their joint payoff equally. Thus, for $i \in S$ we have:

$$
\begin{aligned}
\varphi_{i}\left(c \cdot e^{(S, P)}\right) & =\frac{1}{|S|} \sum_{k \in S} \varphi_{k}\left(c \cdot e^{(S, P)}\right)=-\frac{1}{|S|} \sum_{j \notin S} \varphi_{j}\left(c \cdot e^{(S, P)}\right) \\
& =\frac{1}{|S|} \sum_{j \notin S} \varphi_{j}\left(c \cdot \alpha_{j}(S, P) \cdot e^{\left(S_{+j}, \tau_{j}^{S}(P)\right)}\right) .
\end{aligned}
$$

We observe that formulas (.1) and (.2) provide two recursive equations for $\varphi_{i}\left(c \cdot e^{(S, P)}\right)$ for both cases: $i \in S$ and $i \notin S$. This concludes our proof.

We note that the above proof of Lemma 1 requires only the weaker version of Additivity, i.e., $\varphi\left(v_{1}+v_{2}\right)=\varphi\left(v_{1}\right)+\varphi\left(v_{2}\right)$.

Lemma 2. For every family of quasiprobability distribution $p$, the p-Process Shapley Value satisfies Shapley's axioms.

Proof. We will examine the axioms one by one. First, let us consider Efficiency and recall the formula (7) for the $p$-Process Shapley Value:

$\varphi_{i}^{p}(v)=\frac{1}{|N| !} \sum_{\pi \in \Omega(N)} \sum_{\substack{P \in \mathcal{P}(N) \\\left\{\left\{\pi^{-1}(\mathfrak{m})\right\}\right\} \in P}} p_{n-1}\left(\pi(P)_{-m}\right)\left(v\left(C_{i}^{\pi} \cup\{i\}, P_{\left[C_{i}^{\pi} \cup\{i\}\right]}\right)-v\left(C_{i}^{\pi}, P_{\left[C_{i}^{\pi}\right]}\right)\right)$.

Since, for any permutation $\pi$ and partition $P$, the elementary marginal contributions add up to $v(N,\{N\})$, we have that:

$$
\begin{aligned}
\sum_{i \in N} \varphi_{i}^{p}(v) & =\frac{1}{|N| !} \sum_{\pi \in \Omega(N)} \sum_{\substack{P \in \mathcal{P}(N) \\
\left\{\left\{\pi^{-1}(\mathfrak{m})\right\}\right\} \in P}} p_{n-1}\left(\pi(P)_{-m}\right) \\
& \sum_{i \in N}\left(v\left(C_{i}^{\pi} \cup\{i\}, P_{\left[C_{i}^{\pi} \cup\{i\}\right]}\right)-v\left(C_{i}^{\pi}, P_{\left[C_{i}^{\pi}\right]}\right)\right) \\
& =\frac{1}{|N| !} \sum_{\pi \in \Omega(N)} \sum_{\substack{P \in \mathcal{P}(N) \\
\left\{\left\{\pi^{-1}(\mathfrak{m})\right\}\right\} \in P}} p_{n-1}\left(\pi(P)_{-m}\right) \cdot v(N,\{N\})=v(N,\{N\}),
\end{aligned}
$$

where the last transformation comes from the fact that, for every permutation $\pi$, the probabilities of all partitions sum up to one: $\sum_{P \in \mathcal{P}(N)} p_{n}(\pi(P))=1$ (in the above formula, the sum of probabilities $p_{n-1}$ of all the partitions of the set without the last player from $\pi$ is considered). 
From formula (7), it is clear that the value satisfies Symmetry and Additivity. Regarding Symmetry, it does not favor any player, hence the permutation of coalitions' values will permute payoffs accordingly. The value is additive as $\varphi_{i}^{p}\left(v_{1}+v_{2}\right)$ can be split into two expressions representing $\varphi_{i}^{p}\left(v_{1}\right)$ and $\varphi_{i}^{p}\left(v_{2}\right)$.

Finally, consider the Null-Player Axiom. As the $p$-Process Shapley Value is a weighted average of elementary marginal contributions, if all of them equal zero, then the payoff of a player equals zero as well.

In what follows, we provide the proofs of all the theorems and propositions in the main body of the article.

Theorem 1 (p. 11). The Stochastic Shapley Value is the only value that satisfies Efficiency, Symmetry, Additivity and the CRP-Null-Player Axiom.

Proof. Let us begin by observing that the definition of marginal contribution $m c_{i}^{C R P}(v)$ is a special case of a general formula (9) with the following weights:

$$
\alpha_{i}^{C R P}(S, P)=\frac{|P(i) \backslash\{i\}|}{|N|-|S|},
$$

assuming $|\emptyset|=1$. In particular, the $C R P$-Null-Player Axiom is identical with the corresponding $\alpha^{C R P}$-Null-Player Axiom. Moreover, Lemma 1 implies the uniqueness of the axiomatization: Efficiency, Additivity, Symmetry and CRP-Null-Player Axiom. Now, since Lemma 2 shows that the Stochastic Shapley Value satisfies Efficiency, Additivity and Symmetry, it is enough to show that it also satisfies the $C R P$-Null-Player Axiom. This simply follows from formula (4).

Theorem 2 (p. 15). The value can be obtained using the process approach for a family of quasiprobability distributions $p$ if and only if it satisfies Efficiency, Symmetry, Additivity and the Null-Player Axiom.

Proof. From Lemma 2, we have that every $p$-Process Shapley Value satisfies Shapley's axioms. Now, consider a set of players $N=\{1, \ldots, n\}$ and let $\tilde{\varphi}$ be a value that satisfies Shapley's axioms. We will show that there exists a quasiprobability distribution $p_{n-1}$ such that

$$
\tilde{\varphi}_{i}\left(e^{(S, P)}\right)=\varphi_{i}^{p}\left(e^{(S, P)}\right) \text { for every }(S, P) \in E C, i \notin S,
$$

where $\varphi^{p}$ denotes the $p$-Process Shapley Value. This fact: 
- combined with Efficiency and Symmetry implies that $\tilde{\varphi}_{i}\left(e^{(S, P)}\right)=\varphi_{i}^{p}\left(e^{(S, P)}\right)$ also for $i \in S$; and

- combined with Additivity $\tilde{\varphi}(v)=\varphi^{p}(v)$ for arbitrary game $v$.

Since $\tilde{\varphi}$ and every $p$-Process Shapley Value satisfy Efficiency, Symmetry and Additivity this is enough to prove that both values are equivalent.

We will use the reverse induction on the size of $S$ and prove that there exists a probability distribution $p_{n-1}$ such that the condition from formula (.3) is satisfied for every $(S, P)$ such that $|N|-|S| \leq k$.

The basis for our induction is simple. This is because $\varphi_{i}\left(e^{\left(N_{-i},\left\{N_{-i},\{i\}\right\}\right)}\right)=$ $-\frac{1}{n}$ (which follows from Shapley's axioms) implies that $p_{n-1}(\{\mathbb{1}\})=1$. Now, let us assume that there exists a quasiprobability distribution $\tilde{p}$ such that formula (.3) holds for every $(S, P)$ such that $|N|-|S|<k$. We will prove that there exists $p$ such that formula $(.3)$ holds for every $(S, P)$ such that $|N|-|S|=k$.

Consider formula (8) for the $p$-Process Shapley Value applied to $e^{(S, P)}$ with $i \notin S$ :

$$
\varphi_{i}^{p}\left(e^{(S, P)}\right)=\frac{|S| !}{|N| !} \sum_{\pi \in \Omega(N \backslash S)} p_{n-1}\left(\pi_{+i}(P \backslash S)\right) .
$$

It is clear that $\varphi_{i}^{\tilde{p}}\left(e^{(S, P)}\right)$ for $|N|-|S|=m<k$ depends only on $\tilde{p}_{n-1}\left(P_{m}\right)$ where $P_{m} \in\{\mathbb{1}, 2, \ldots, m\}$. Thus, we will show that there exists a quasiprobability distribution $p_{n-1}$ that satisfy the following two conditions:

(a) for each partition smaller than $k$ elements $p_{n-1}$ is equal to $\tilde{p}_{n-1}$ (i.e., $p_{n-1}\left(P_{m}\right)=\tilde{p}_{n-1}\left(P_{m}\right)$ for every $P_{m} \in\{\mathbb{1}, \ldots, \mathrm{m}\}$ where $\left.m<k\right)$; and

(b) values $p_{n-1}\left(P_{k}\right)$ of partitions of size $k$ are such that $\tilde{\varphi}_{i}\left(e^{(S, P)}\right)=\varphi_{i}^{p}\left(e^{(S, P)}\right)$ is satisfied for $(S, P)$ with $|N|-|S|=k$.

Let $P_{k-1} \in \mathcal{P}(\{\mathbb{1}, \ldots, \mathbb{k}-\mathbb{1}\})$ be an arbitrary partition of $k-1$ elements. To meet condition (a), based on the recursive formula (6) for quasiprobability distributions, we get that values of $p_{n-1}$ for all partitions of size $k$ created by adding element $\mathbb{k}$ to $P_{k-1}$ have to sum up to $\tilde{p}_{n-1}\left(P_{k-1}\right)$ :

$$
\sum_{\substack{P_{k} \in \mathcal{P}(\{\mathbb{1}, \ldots, \mathbb{k}\}) \\\left(P_{k}\right)_{-k}=P_{k-1}}} p_{n-1}\left(P_{k}\right)=\sum_{S \in P_{k-1} \cup\{\emptyset\}} p_{n-1}\left(P_{k-1} \backslash S \cup\{S \cup \mathbb{k}\}\right)=\tilde{p}_{n-1}\left(P_{k-1}\right) .
$$


Let us consider requirements based on condition (b). Let $P_{k} \in \mathcal{P}(\{\mathbb{1}, \ldots, \mathbb{k}\})$ be an arbitrary partition of $k$ elements. For $P_{k}$, we construct the corresponding simple game $e^{(S, P)}$, denoted $w\left(P_{k}\right)$, with $S=\{k+1, \ldots, n\}$ and players $\{1, \ldots, k\}$ partitioned as elements $\{\mathbb{1}, \ldots, \mathbb{k}\}$ in $P_{k}$ :

$$
w\left(P_{k}\right)=e^{\left(\{k+1, \ldots, n\},\left(\pi_{i d}^{-1}\left(P_{k}\right) \cup\{k+1, \ldots, n\}\right)\right)} .
$$

Now, formula (.4) applied for player $k$ in game $w\left(P_{k}\right)$ yields the following condition on $p_{n-1}$ :

$$
\sum_{\pi \in \Omega(\{1, \ldots, k-1\})} p_{n-1}\left(\pi_{+k}\left(P_{k}\right)\right)=\frac{n !}{(n-k) !} \cdot\left(-\tilde{\varphi}_{k}\left(w\left(P_{k}\right)\right)\right) .
$$

Note that based on Symmetry, the players' names do not matter, thus every simple game $e^{(S, P)}$ with $|N|-|S|=k$ is equivalent to $w\left(P_{k}\right)$ for some $P_{k} \in$ $\mathcal{P}(\{\mathbb{1}, \ldots, \mathbb{k}\})$. Therefore, satisfying formula (.6) for every $P_{k}$ is necessary and sufficient to meet condition (b).

Let us summarize the above conditions on $p_{n-1}$ for partitions associated with a given $P_{k-1} \in \mathcal{P}(\{1, \ldots, k-1\})$. Let $Q=\left\{\pi\left(P_{k-1}\right) \mid \pi \in \Omega(\{1, \ldots, k-\right.$ $1\})\}$ be the set of different partitions resulting from permuting the elements in $P_{k-1}$, and $\Omega_{Q}$ the corresponding set of permutations of $\{1, \ldots, k-1\}$ that result in partitions from $Q: \Omega_{Q} \subseteq \mathcal{P}(\{1, \ldots, k-1\})$ such that $\left|\Omega_{Q}\right|=|Q|$ and $\pi_{1}, \pi_{2} \in \Omega_{Q} \Rightarrow \pi_{1}\left(P_{k-1}\right) \neq \pi_{2}\left(P_{k-1}\right)$. Adding element $k$ to each of partitions $\Omega$ results in $\left|P_{k-1}\right|+1$ different partitions $P_{k}$. Each such partition can be characterized by a pair $(\pi, S) \in\left(\Omega_{Q} \times\left(P_{k-1} \cup \emptyset\right)\right)$ where $S$ denotes a coalition in which $k$ is added:

$$
a(\pi, S)=p_{n-1}\left(\pi_{+k}\left(P_{k-1} \backslash S \cup\{S \cup\{\mathbb{k}\}\}\right) .\right.
$$

We will analyze the conditions on these values imposed by formulas (.5) and (.6).

Formula (.5) yields that for every $\pi \in \Omega_{Q}$ we have:

$$
\sum_{S \in P_{k-1} \cup\{\emptyset\}} a(\pi, S)=\tilde{p}_{n-1}\left(\pi\left(P_{k-1}\right)\right) .
$$

Furthermore, from formula (.6), we have for every $S \in P_{k-1} \cup\{\emptyset\}$ :

$$
\sum_{\pi \in \Omega_{Q}}\left(\sum_{\substack{\pi_{2} \in \Omega(\{1, \ldots, k-1\}) \\ \pi_{2}\left(P_{k-1}\right)=\pi\left(P_{k-1}\right)}} 1\right) a(\pi, S)=\frac{n !}{(n-k) !} \cdot\left(-\tilde{\varphi}_{k}\left(w\left(P_{k-1} \backslash S \cup\{S \cup \mathbb{k}\}\right)\right)\right) .
$$


As the number of permutations $\pi_{2}$ which gives the same partition as $\pi$ applied to $P_{k-1}$ does not depend on $\pi$ (and equal $\prod_{T \in P_{k-1}}|T|$ !), we get

$$
\sum_{\pi \in \Omega_{Q}} a(\pi, S)=\frac{n !}{(n-k) ! \cdot\left(\prod_{T \in P_{k-1}}|T| !\right)} \cdot\left(-\tilde{\varphi}_{k}\left(w\left(P_{k-1} \backslash S \cup\{S \cup \mathbb{k}\}\right)\right)\right) .
$$

Hence, we have a matrix of variables $\left(\Omega_{Q} \times\left(P_{k-1} \cup \emptyset\right)\right)$ and the conditions imply what is the sum of each row and each column. To prove there exists a solution, it is enough to observe that this system of equations is not contradictory, i.e, the sum of rows equals the sum of columns:

$$
\begin{aligned}
\sum_{\pi \in \Omega_{Q}} \tilde{p}_{n-1}\left(\pi\left(P_{k-1}\right)\right) & =\sum_{S \in P_{k-1} \cup\{\emptyset\}} \frac{-n ! \cdot \tilde{\varphi}_{k}\left(w\left(P_{k-1} \backslash S \cup\{S \cup \mathbb{R}\}\right)\right)}{(n-k) ! \cdot\left(\prod_{T \in P_{k-1}}|T| !\right)} \\
\frac{(n-k) !}{n !} \sum_{\pi \in \Omega(\{1, \ldots, k-1\})} \tilde{p}_{n-1}\left(\pi\left(P_{k-1}\right)\right) & =\sum_{S \in P_{k-1} \cup\{\emptyset\}}-\tilde{\varphi}_{k}\left(w\left(P_{k-1} \backslash S \cup\{S \cup \mathbb{k}\}\right)\right) \\
\frac{(n-k) !}{n !} \sum_{\pi \in \Omega(\{1, \ldots, k-1\})} \tilde{p}_{n-1}\left(\pi\left(P_{k-1}\right)\right) & =\tilde{\varphi}_{k}\left(w\left(P_{k-1}\right)\right) \\
\varphi_{k}^{p}\left(w\left(P_{k-1}\right)\right) & =\tilde{\varphi}_{k}\left(w\left(P_{k-1}\right)\right),
\end{aligned}
$$

where we used the Null-Player Axiom. The final equality comes from the inductive assumption. This concludes the proof.

Theorem 3 (p. 15). The value can be obtained using the process approach for a family of probability distributions $p$ if and only if it satisfies Efficiency, Symmetry, Additivity, the Null-Player Axiom, and (Weak) Monotonicity.

Proof. (Weak) Monotonicity states that increasing an elementary marginal contribution of a player does not decrease her payoff. For additive values, this fact is equivalent to the fact that $\varphi_{i}\left(e^{(S, P)}\right) \geq 0$ if $i \in S$, and that $\varphi_{i}\left(e^{(S, P)}\right) \leq 0$ if $i \notin S$ for every $(S, P) \in E C(N)$.

We use the construction from the proof of Theorem 2. We will argue, that, if probability (not quasiprobability) distribution $\tilde{p}_{n-1}$ satisfies formula (.3) for every $(S, P)$ such that $|N|-|S|<k$, then we can define $p_{n-1}$ so that this formula holds also for every $(S, P)$ such that $|N|-|S|=k$.

To this end, we define $a(\pi, S)$ as follows:

$$
a(\pi, S)=\tilde{p}_{n-1}\left(\pi\left(P_{k-1}\right)\right) \cdot \frac{-\tilde{\varphi}_{k}\left(w\left(P_{k-1} \backslash S \cup(S \cup\{\mathbb{k}\})\right)\right)}{\tilde{\varphi}_{k}\left(w\left(P_{k-1}\right)\right)},
$$


if $\tilde{\varphi}_{k}\left(w\left(P_{k-1}\right)\right) \neq 0$, and $a(\pi, S)=0$, otherwise. These values are nonnegative for monotonic $\tilde{v}$. It is easy to check that they satisfy all conditions: if $\tilde{\varphi}_{k}\left(w\left(P_{k-1}\right)\right) \neq 0$, then

$$
\sum_{S \in P_{k-1} \cup\{\emptyset\}} a(\pi, S)=\tilde{p}_{n-1}\left(\pi\left(P_{k-1}\right)\right)
$$

from the Null-Player Axiom, and

$$
\sum_{\pi \in \Omega(\{1, \ldots, k-1\})} a(\pi, S)=\frac{n !}{(n-k) !} \cdot\left(-\tilde{\varphi}_{k}\left(w\left(P_{k-1} \backslash S \cup(S \cup\{\mathbb{k}\})\right)\right)\right)
$$

since $\tilde{\varphi}_{k}\left(w\left(P_{k-1}\right)\right)=\varphi^{\tilde{p}}\left(w\left(P_{k-1}\right)\right)=\frac{(n-k) !}{n !} \sum_{\pi \in \Omega(\{1, \ldots, k-1\})} p_{n-1}\left(\pi\left(P_{k-1}\right)\right)$. On the other hand, if $\tilde{\varphi}_{k}\left(w\left(P_{k-1}\right)\right)=0$, then all values $p_{n-1}\left(\pi\left(P_{k-1}\right)\right)$ must equal zero, and non-negative (from monotonicity) values of $\left(-\tilde{\varphi}_{k}\left(w\left(P_{k-1} \backslash S \cup(S \cup\right.\right.\right.$ $\{\mathbb{k}\}))$ ) must all equal zero. Thus, $a(\pi, S)=0$ for all values satisfies the conditions. This concludes the proof.

Theorem 4 (p. 17). There exists a unique value that satisfies Efficiency, Symmetry, Additivity and the $\alpha$-Null-Player Axiom for every $\alpha$, and this value is the $p^{\alpha}$-Process Shapley Value, where $p^{\alpha}$ is defined as follows:

$$
p_{n}^{\alpha}(P)=\prod_{i \in\{1, \ldots, n\}} \alpha_{i}\left(\{i+1, \ldots, n, n+1\},\left(\pi_{i d}^{-1}(P) \cup\{n+1\}\right)_{[\{i+1, \ldots, n, n+1\}]}\right),
$$

for every $P \in \mathcal{P}(\{\mathbb{1}, \ldots, n\})$. Moreover, for quasiprobability distribution $p$, there exists $\alpha$ such that the $p$-Process Shapley Value is the value resulting from $\alpha$-parameterized Shapley's axiomatization if and only if $p$ satisfies zeroconsistency, i.e.,

$$
\sum_{\pi \in \Omega(\{\mathbb{1}, \ldots, \mathbb{k}-\mathbb{1}\})} p_{n}\left(\pi\left(\left(P_{k}\right)_{-\mathbb{k}}\right)\right)=0 \Rightarrow \sum_{\pi \in \Omega(\{\mathbb{1}, \ldots, \mathbb{k}-\mathbb{1}\})} p_{n}\left(\pi_{+\mathbb{k}}\left(P_{k}\right)\right)=0 .
$$

for every $n, k \leq n$, and $P_{k} \in \mathcal{P}(\{\mathbb{1}, \ldots, \mathbb{k}\})$.

Proof. In Lemma 1, we have already proved the uniqueness of the axiomatization. Thus, to prove the first part of the theorem it is enough to show that the $p^{\alpha}$-Process Shapley Value with $p^{\alpha}=\left(p_{1}^{\alpha}, p_{2}^{\alpha}, \ldots\right)$ defined as in formula (.7) satisfies all four axioms. In Lemma 2, we have already proved that every $p$-Process Shapley Value satisfies Efficiency, Additivity and Symmetry. 
To see that the $p^{\alpha}$-Process Shapley Value satisfies the $\alpha$-Null-Player Axiom let us recall its definition by formula (8):

$$
\begin{array}{r}
\varphi_{i}^{p}(v)=\sum_{\substack{(S, P) \in E C \\
i \in S}} \frac{(|S|-1) !}{|N| !} \sum_{\pi \in \Omega(N \backslash S)} \sum_{T \in P \backslash S \cup\{\emptyset\}} p_{n-1}\left(\pi_{+i}\left(\tau_{i}^{T}(P) \backslash S_{-i}\right)\right) \\
\left(v(S, P)-v\left(S_{-i}, \tau_{i}^{T}(P)\right)\right) .
\end{array}
$$

Since, from the definition of $p^{\alpha}$, we have that:

$$
p_{n-1}^{\alpha}\left(\pi_{+i}\left(\tau_{i}^{T}(P) \backslash S_{-i}\right)\right)=p_{n-1}^{\alpha}(\pi(P \backslash S)) \cdot \alpha_{i}\left(S_{-i}, \tau_{i}^{T}(P)\right)
$$

then we can reformulate the above formula for $\varphi_{i}^{p}(v)$ as follows:

$$
\begin{aligned}
& \varphi_{i}^{p}(v)= \sum_{\substack{S, P) \in E C \\
i \in S}} \frac{(|S|-1) !}{|N| !} \sum_{\pi \in \Omega(N \backslash S)} p_{n-1}^{\alpha}(\pi(P \backslash S)) \cdot \\
& \sum_{\substack{T \in P \backslash S \cup\{\emptyset\} \\
=}} \alpha_{i}\left(S_{-i}, \tau_{i}^{T}(P)\right)\left(v(S, P)-v\left(S_{-i}, \tau_{i}^{T}(P)\right)\right) \\
& \sum_{(S, P) \in E C} \frac{(|S|-1) !}{|N| !} \sum_{i \in S} p_{n-1}^{\alpha}(\pi(P \backslash S)) \cdot\left(m c_{i}^{\alpha}(v)\right)(S, P),
\end{aligned}
$$

where we follow the definition of the general form of marginal contribution, i.e., formula (9). Thus, the $\alpha$-Null-Player Axiom is satisfied - if all $\alpha$-marginal contributions equal zero, then the payoff of a player equals zero as well.

In the second part of the proof, we first argue that if $p$-Process Shapley Value can be obtained using marginality approach, then $p$ satisfies zeroconsistency. Assume that the value meets the $\alpha$-Null-Player Axiom for some $\alpha$. For every embedded coalition $(S, P) \in E C$ such that $i \notin S$ we get:

$$
\varphi_{i}\left(\alpha_{i}(S, P) \cdot e^{\left(S_{+i}, \tau_{i}^{S}(P)\right)}\right)=\varphi_{i}\left(e^{(S, P)}\right) .
$$

Thus, if $\varphi_{i}\left(e^{\left(S_{+i}, \tau_{i}^{S}(P)\right)}\right)=0$, then $\varphi_{i}\left(e^{(S, P)}\right)=0$. This fact combined with formula (8) for the $p$-Process Shapley Value gives exactly the zero-consistency condition on $p$.

Now, we prove that this condition is sufficient, i.e., that for every $p$ Process Shapley Value such that $p$ satisfies zero-consistency there exists $\alpha$ so 
that the $p$-Process Shapley Value is the unique value which satisfies Efficiency, Additivity, Symmetry and the $\alpha$-Null-Player Axiom. To this end, consider a family of quasiprobability distributions $p$, and let us define $\alpha(p)$ as follows:

$$
\alpha(p)_{i}(S, P)=\frac{\sum_{\pi \in \Omega(N \backslash(S \cup\{i\}))} p_{n-1}\left(\pi_{+i}(P \backslash S)\right)}{\sum_{\pi \in \Omega(N \backslash(S \cup\{i\}))} p_{n-1}\left(\pi\left(P_{-i} \backslash S\right)\right)},
$$

if $\sum_{\pi \in \Omega(N \backslash(S \cup\{i\}))} p_{n}\left(\pi\left(P_{-i} \backslash S\right)\right) \neq 0$. Otherwise, $\alpha(p)_{i}(S, P)$ can be defined arbitrarily, as we will argue that they do not matter (e.g., $\alpha(p)_{i}(S, P)=1$ iff $\{i\} \in P$, and $\alpha(p)_{i}(S, P)=0$, otherwise). We argue that $\alpha(p)$ are proper weights in the marginality approach, i.e., they are symmetrical and sum up to one. The former property is obvious. To see the later one, for arbitrary embedded coalition $(S, P) \in E C$ such that $i \in S$ we calculate:

$$
\begin{aligned}
\sum_{T \in P \backslash\{S\} \cup\{\emptyset\}} \alpha(p)_{i}\left(S_{-i}, \tau_{i}^{T}(P)\right) & =\frac{\sum_{\pi \in \Omega(N \backslash S)} \sum_{T \in P \backslash S \cup\{\emptyset\}} p_{n}\left(\pi_{+i}\left(\tau_{i}^{T}(P) \backslash S_{-i}\right)\right)}{\sum_{\pi \in \Omega(N \backslash S)} p_{n}(\pi(P \backslash S))} \\
& =\frac{\sum_{\pi \in \Omega(N \backslash S)} p_{n}(\pi(P \backslash S))}{\sum_{\pi \in \Omega(N \backslash S)} p_{n}(\pi(P \backslash S))}=1,
\end{aligned}
$$

where we used formula (6).

Now, we show that these weights leads to the $p$-Process Shapley Value. Lemma 2 states that every $p$-Process Shapley Value meets Efficiency, Additivity and Symmetry; hence, it is enough to consider only the $\alpha(p)$-Null-Player Axiom. To this end, consider a game:

$$
\tilde{v}=\alpha(p)_{i}(S, P) \cdot e^{\left(S_{+i}, T_{i}^{S}(P)\right)}+e^{(S, P)}
$$

for $(S, P)$ such that $i \notin S$. Note that player $i$ is a $\alpha(p)$-null-player in game $\tilde{v}$. From Additivity it is enough to show that the payoff of player $i$ in such a game for an arbitrary embedded coalition equals zero.

From formula (8) for the $p$-Process Shapley Value we have:

$$
\begin{aligned}
\varphi_{i}^{p}(\tilde{v})=\frac{|S| !}{|N| !} \cdot \alpha(p)_{i}(S, P) \sum_{\pi \in \Omega(N \backslash(S \cup\{i\}))} & p_{n-1}\left(\pi\left(P_{-i} \backslash S\right)\right) \\
& -\frac{|S| !}{|N| !} \sum_{\pi \in \Omega(N \backslash(S \cup\{i\}))} p_{n-1}\left(\pi_{+i}(P \backslash S)\right) .
\end{aligned}
$$


If $\sum_{\pi \in \Omega(N \backslash(S \cup\{i\}))} p_{n-1}\left(\pi\left(P_{-i} \backslash S\right)\right)$ equals 0 , then zero-consistency implies that the sum $\sum_{\pi \in \Omega(N \backslash(S \cup\{i\}))} p_{n-1}\left(\pi_{+i}(P \backslash S)\right)$ also equals zero. Otherwise, a reformulation gives

$\varphi_{i}^{p}(\tilde{v})=\frac{|S| !}{|N| !}\left(\sum_{\pi \in \Omega(N \backslash(S \cup\{i\}))} p_{n-1}\left(\pi_{+i}(P \backslash S)\right)-\sum_{\pi \in \Omega(N \backslash(S \cup\{i\}))} p_{n-1}\left(\pi_{+i}(P \backslash S)\right)\right)$

which simplifies to zero. This concludes the proof. 